JOM 23398

\title{
Structural characterization and comparison of $\left[\mathrm{Ru}_{2}\left(\mathrm{O}_{2} \mathrm{CR}\right)_{2}(\mathrm{CO})_{4} \mathrm{~L}_{2}\right]$
}

\author{
Kom-Bei Shiu \\ Department of Chemistry, National Cheng Kung University, Tainan 70101 (Taiwan) \\ Shie-Ming Peng and Ming-Chu Cheng \\ Department of Chemistry, National Taiwan University, Taipei 10764 (Taiwan)
}

(Received October 9, 1992; in revised form November 20, 1992)

\begin{abstract}
The structures of $\left[\mathrm{Ru}_{2}(\mathrm{CO})_{4}\left(\mathrm{O}_{2} \mathrm{CR}\right)_{2}\left(\mathrm{PPh}_{3}\right)_{2}\right]\left(\mathrm{R}=\mathrm{Me}(1)\right.$, $\mathrm{Ph}(2)$, and $\left.\mathrm{CF}_{3}(3)\right)$ have been determined by $\mathrm{X}$-ray crystallography: 1 , monoclinic, $C 2 / c, a=24.630(8), b=9.2476(21), c=18.735(4) \AA, \beta=101.710(20)^{\circ}, Z=4, R=0.026, R_{\mathrm{w}}=0.025$, based on 3243 reflections with $I>2 \sigma(I)$; 2, orthorhombic, $P 2_{1} 2_{1} 2_{1}, a=12.590(3), b=18.188(3), c=20.804(5) \AA, Z=4, R=0.033, R_{w}=0.026$, based on 3410 reflections with $I>2 \sigma(I) ; 3$, triclinic, $P \overline{1}, a=12.7329(19), b=13.450(4), c=13.7939(20) \AA, a=76.770(16)$, $\beta=73.305(14), \gamma=76.017(16)^{\circ}, Z=2, R=0.054, R_{w}=0.047$, based on 5543 reflections with $I>2 \sigma(I)$. The Ru-Ru bond lengths are $2.7360(9) \AA$ in $1,2.7411(8) \AA$ in 2, and 2.7276(9) $\AA$ in 3. Comparison of these structures with those of other dinuclear $R u^{1}$ structures of the general formula $\left[\mathrm{Ru}_{2}\left(\mathrm{O}_{2} \mathrm{CR}\right)_{2}(\mathrm{CO})_{4} \mathrm{~L}_{2}\right]\left(\mathrm{L}=\mathrm{CO} . \mathrm{PPh}_{3}, \mathrm{PBu}_{3}, \mathrm{py}, \mathrm{H}_{2} \mathrm{O}\right.$, or $\mathrm{PhCO} \mathrm{C}_{2} \mathrm{H}=\mathrm{Re}, \mathrm{CF}_{3},{ }^{\mathrm{n}} \mathrm{Pr}$, or 4-FC $\left.{ }_{6} \mathrm{H}_{4}\right)$ or of $\left[\mathrm{Ru}_{2}\left(\mathrm{O}_{2} \mathrm{CPh}\right)_{2}(\mathrm{CO})_{4}\right]_{\infty}$ reported to date leads to the conclusion that the axial ligand is the most influential in determining the length of the $\mathrm{Ru}-\mathrm{Ru}$ single bond, and that the equatorial ligand is responsible for the secondary effect.
\end{abstract}

\section{Introduction}

Our recent interest in exploring the unusual physical and chemical influences of multidentate ligands on the transition-metal carbonyl derivatives of the ligands [1] has led us to investigate the influence of bidentate ligands such as carboxylates in dinuclear carbonyl complexes. Since the complexes such as $\left[\mathrm{Ru}_{2}\left(\mathrm{O}_{2} \mathrm{CR}\right)_{2}\right.$ $(\mathrm{CO})_{4} \mathrm{~L}_{2}$ ] can be viewed as possible intermediates interconverting for the low- and high-valent cluster compounds (LVCs and HVCs), defined in 1983 by Cotton [2], research in this direction might identify new synthetic routes connecting the numerous LVCs such as $\left[\mathrm{Ru}_{3}(\mathrm{CO})_{12}\right.$ ] [3] and the well-established, multiply bonded dimers such as $\left[\mathrm{Ru}_{2}\left(\mathrm{O}_{2} \mathrm{CR}\right)_{4} \mathrm{Cl}\right]$ [4]. In this paper, we present the structural details of $\left[\mathrm{Ru}_{2}\left(\mathrm{O}_{2}\right.\right.$ $\left.\mathrm{CR})_{2}(\mathrm{CO})_{4}\left(\mathrm{PPh}_{3}\right)_{2}\right] \mathrm{R}=\mathrm{Me}, \mathrm{Ph}$, or $\left.\mathrm{CF}_{3}\right)$, compounds which are known to be important catalysts or catalytic precursors in reactions such as the hydroformylation of olefins or the addition of carboxylic acids to alkynes

Correspondence to: Dr. K.-B. Shiu.
[5-9], and we compare them with those of published dimers with different axial ligands, $L$, with $L=$ pyridine (py) or CO [10,11]. Similar comparisons in HVCs such as $\left[\mathrm{M}_{2}\left(\mathrm{O}_{2} \mathrm{CR}\right)_{4} \mathrm{~L}_{2}\right](\mathrm{M}=\mathrm{Mo}, \mathrm{Cr}$, or $\mathrm{Rh})$ [12] have been reported in the literature. We hope that the comparison in $\left[\mathrm{Ru}_{2}\left(\mathrm{O}_{2} \mathrm{CR}\right)_{2}(\mathrm{CO})_{4} \mathrm{~L}_{2}\right]$ can improve our understanding of the activation of the metal-metal multiply bonded dimers, the metal-metal bonds being maintained, and of the conversion from HVCs into LVCs.

\section{Experimental section}

Compounds, $\left[\mathrm{Ru}_{2}\left(\mathrm{O}_{2} \mathrm{CR}\right)_{2}(\mathrm{CO})_{4}\left(\mathrm{PPh}_{3}\right)_{2}\right](\mathrm{R}=\mathrm{Me}$, 1; Ph, 2; or $\mathrm{CF}_{3}, 3$ ), were prepared by the published procedure $[9,13] .{ }^{31} \mathrm{P}\left({ }^{1} \mathrm{H}\right\}$ NMR spectra of $1-3$ in $\mathrm{CDCl}_{3}$ were obtained at room temperature by a Bruker AMX400 FT NMR spectrometer at $162 \mathrm{MHz}$ with $\mathrm{PPh}_{3}$ as an internal standard: 19.8, 21.7 and $21.3 \mathrm{ppm}$, respectively. Single crystals of 1-3 were grown from $\mathrm{CH}_{2} \mathrm{Cl}_{2}$ /hexane at room temperature. The pertinent crystal data are summarized in Table 1 . The methods used have been presented elsewhere [14]. In 1, a $C_{2}$ axis is imposed crystallographically at the center of the 
$\mathrm{Ru}-\mathrm{Ru}$ bond while in $\mathbf{3}$, two $\mathrm{CF}_{3}$ groups are disordered and eight fluorine atom positions with occupancy of 0.75 were found. All non-hydrogen atoms (excepting the fluorine atoms in 3) were refined anisotropically. The final refinement converged smoothly and no chemically significant peaks were found in the final difference maps.

The fractional atomic coordinates of all the non-hydrogen atoms are listed in Table 2. Table 3 contains selected bond lengths and angles for 1-3. Tables of the remaining bond lengths and angles, the hydrogen atomic coordinates, the anisotropic displacement coefficients, and structure factor $\left(F_{\mathrm{o}} v s . F_{\mathrm{c}}\right)$ are available from the authors. The ORTEP plots for 1-3 are drawn in Figs. 1-3, respectively, with the relevant numbering scheme.

\section{Results and discussion}

As shown in Figs. 1-3, compounds 1-3 are quite similar, each adopting a sawhorse or cis-eclipsed struc- ture, in which the two carbonyl groups on each metal are mutually cis and opposite the bridging groups, with the phosphine ligands in the axial sites opposite the $\mathrm{Ru}-\mathrm{Ru}$ bond. This structural feature is similar to that reported for other bridged compounds, $\left[\mathrm{Ru}_{2}\left(\mathrm{O}_{2} \mathrm{CR}\right)_{2}\right.$ $(\mathrm{CO})_{4} \mathrm{~L}_{2}$ ] with $\mathrm{L}=\mathrm{py}, \mathrm{CO}, \mathrm{P}^{t} \mathrm{Bu}_{3}, \mathrm{H}_{2} \mathrm{O}$, or $\mathrm{PhCO}_{2} \mathrm{H}$ and $\mathrm{R}=\mathrm{Me}$, ${ }^{\mathrm{n}} \mathrm{Pr}, 4-\mathrm{F}-\mathrm{C}_{6} \mathrm{H}_{4}$ or $\mathrm{Ph}[10,11,15,16]$ and $\left[\mathrm{Ru}_{2} \mathrm{X}_{2}(\mathrm{CO})_{4}\left(\mathrm{PR}_{3}\right)_{2}\right]$ with $\mathrm{X}^{-}=\mathrm{Cl}^{-}$, 3,5-dimethylpyrazolato $\left(\mathrm{Pz}^{\prime-}\right)$, or 2-oxypyridinato $\left(\mathrm{NC}_{5} \mathrm{H}_{4} \mathrm{O}^{-}\right) ; \mathrm{R}=\mathrm{Ph}$, ${ }^{t} \mathrm{Bu}$, or $p$-tol [17-19] but different from the cisstaggered structure observed for the unbridged dinuclear compound, $\left[\mathrm{Ru}_{2}\left(\mathrm{HBPz}_{3}\right)_{2}(\mathrm{CO})_{4}\right](\mathrm{Pz}=$ pyrazolato) $[20]$.

The Ru-Ru length is $2.7360(9) \AA$ for $1,2.7411(8) \AA$ for 2 , and $2.7276(9) \AA$ for 3 (Table 3 ). The second value is the longest $R u-R u$ distance so far seen in bridged $R u^{I}-R u^{I}$ compounds $[10,11,15-19]$, though that in the unbridged compound, $\left[\mathrm{Ru}_{2}\left(\mathrm{HBPz}_{3}\right)_{2}(\mathrm{CO})_{4}\right]$ [20] is even longer (2.882(1) $\AA$ ). In order to understand the possible factors influencing the variation in $\mathbf{R u}-\mathbf{R u}$

TABLE 1. Crystal data for 1-3

\begin{tabular}{|c|c|c|c|}
\hline compound & 1 & 2 & 3 \\
\hline $\begin{array}{l}\text { empirical formula } \\
\text { color } \\
\text { crystal size (mm) }\end{array}$ & $\begin{array}{l}\mathrm{C}_{44} \mathrm{H}_{38} \mathrm{O}_{8} \mathrm{P}_{2} \mathrm{Ru}_{2} \\
\text { yellow } \\
0.40 \times 0.40 \times 0.45\end{array}$ & $\begin{array}{l}\mathrm{C}_{54} \mathrm{H}_{40} \mathrm{O}_{8} \mathrm{P}_{2} \mathrm{Ru}_{2} \\
\text { orange yellow } \\
0.30 \times 0.40 \times 0.40\end{array}$ & $\begin{array}{l}\mathrm{C}_{44} \mathrm{H}_{30} \mathrm{~F}_{6} \mathrm{O}_{8} \mathrm{P}_{2} \mathrm{Ru}_{2} \\
\text { orange yellow } \\
0.40 \times 0.40 \times 0.30\end{array}$ \\
\hline $\begin{array}{l}\text { space group } \\
\text { unit cell dimensions }\end{array}$ & monoclinic. $C 2 / c$ & orthorhombic, $P 2_{1} 2_{1} 2_{1}$ & triclinic, $P \overline{1}$ \\
\hline$a, b, c, \AA$ & $24.630(8), 9.2476(21), 18.735(4)$ & $12.590(3), 18.188(3), 20.804(5)$ & $12.7329(19), 13.450(4), 13.7936(20)$ \\
\hline$\alpha, \beta, \gamma, \operatorname{deg}$ & $90,101.710(20), 90$ & $90,90,90$ & $76.770(16), 73.305(14), 76.017(16)$ \\
\hline volume $\stackrel{\circ}{ }^{3}$ & $4178.4(18)$ & 4764.1(17) & $2163.1(7)$ \\
\hline$Z$ & 4 & 4 & 2 \\
\hline Formula weight & 958.75 & 1080.99 & 1064.8 \\
\hline$D_{\text {calc }}, \mathrm{g} / \mathrm{cm}^{3}$ & 1.524 & 1.507 & 1.635 \\
\hline no. of orientatn rflns & 24 & 26 & 24 \\
\hline $2 \theta$ range, $\mathrm{deg}$ & $18.32-24.52$ & $18.74-23.42$ & $18.70-22.56$ \\
\hline $\begin{array}{l}h, k, l \text { ranges } \\
\text { abs cor }\end{array}$ & -29 to 28,0 to 10,0 to 22 & $\begin{array}{l}0 \text { to } 14,0 \text { to } 21,0 \text { to } 24 \\
\Psi \text { scan }\end{array}$ & -14 to 15,0 to $15,-15$ to 16 \\
\hline abs coeff; $\mathrm{mm}^{-1}$ & 1.60 & 0.73 & 0.83 \\
\hline transm range & $0.9515-0.9947$ & $0.9503-0.9993$ & $0.9377-0.9993$ \\
\hline $\begin{array}{l}F(000) \\
\text { diffractometer used }\end{array}$ & 2319.78 & $\begin{array}{l}1928 \\
\text { Nonius CAD4 }\end{array}$ & 1060 \\
\hline radiation; $\lambda, \AA$ & & Mo $\mathrm{K} \alpha ; 0.70930$ & \\
\hline temperature $(\mathbf{K})$ & & 297 & \\
\hline scan type & & $\theta / 2 \theta$ & \\
\hline $2 \theta$ range, deg & & $2-50$ & \\
\hline $\begin{array}{l}\text { scan speed, deg/min } \\
\text { std rflns }\end{array}$ & $16.48 / 2-16.48 / 7$ & $\begin{array}{l}16.48 / 2-16.48 / 9 \\
3 \mathrm{std} / 7200 \mathrm{sec}\end{array}$ & $16.48 / 2-16.48 / 10$ \\
\hline decay; $\%$ & $\leq 1$ & $\leq 2$ & $\leq 2$ \\
\hline no. of unique rflns & 3669 & 4635 & 7583 \\
\hline no. of rflns $\left(N_{\mathrm{o}}\right)$ used & 3243 with $I>2.0 \sigma(I)$ & 3410 with $I>2.0 \sigma(I)$ & 5543 with $I>2.0 \sigma(I)$ \\
\hline no. of atoms refined & 47 & 106 & 94 \\
\hline no. of params $\left(N_{\mathrm{v}}\right)$ refined & 254 & 596 & 538 \\
\hline $\max \Delta / \sigma$ ratio & 0.061 & 0.181 & 0.280 \\
\hline$R, R_{\omega} \mathrm{S}^{\mathrm{a}}$ & $0.0264,0.025,2.74$ & $0.033,0.026,1.43$ & $0.054,0.047,3.16$ \\
\hline resid peak; hole, $\mathrm{e} / \AA^{3}$ & $0.30,-0.46$ & $1.46,-1.78^{b}$ & $1.78,-1.16^{b}$ \\
\hline
\end{tabular}

${ }^{\mathrm{a}} \mathrm{S}=\left[\sum \omega\left|F_{\mathrm{o}}-F_{\mathrm{c}}\right|^{2} /\left(N_{\mathrm{o}}-N_{\mathrm{v}}\right)\right]^{1 / 2}, \omega^{-1}=\sigma^{2}\left(F_{\mathrm{o}}\right){ }^{\mathrm{b}}$ Ghost peaks. 
TABLE 2. Fractional atomic coordinates and $B_{\text {iso }}{ }^{a}$ for 1-3

\begin{tabular}{lllll}
\hline \multicolumn{2}{c}{$x$} & $y$ & \multicolumn{3}{l}{$l$} & $B_{\text {iso }}$ \\
\hline (a) Compound 1 & & & \\
Ru & $0.449381(9)$ & $0.44438(3)$ & $0.203545(13)$ & $2.135(10)$ \\
P & $0.36479(3)$ & $0.37055(9)$ & $0.11831(4)$ & $2.24(3)$ \\
O1 & $0.48535(8)$ & $0.27780(23)$ & $0.15177(11)$ & $3.28(10)$ \\
O2 & $0.43112(8)$ & $0.28018(24)$ & $0.27477(11)$ & $3.54(11)$ \\
C1 & $0.53247(12)$ & $0.2284(3)$ & $0.17213(16)$ & $2.84(14)$ \\
C2 & $0.54932(15)$ & $0.1019(4)$ & $0.13206(21)$ & $5.46(20)$ \\
C3 & $0.47073(12)$ & $0.5828(4)$ & $0.14496(17)$ & $3.20(14)$ \\
O3 & $0.48409(10)$ & $0.6706(3)$ & $0.10862(14)$ & $5.60(13)$ \\
C4 & $0.42082(11)$ & $0.5893(3)$ & $0.25191(17)$ & $3.05(14)$ \\
O4 & $0.40372(9)$ & $0.6825(3)$ & $0.28150(13)$ & $4.74(12)$ \\
C11 & $0.35293(12)$ & $0.4635(3)$ & $0.03085(15)$ & $2.55(13)$ \\
C12 & $0.39717(13)$ & $0.4707(4)$ & $-0.00579(17)$ & $3.49(16)$ \\
C13 & $0.39118(15)$ & $0.5374(4)$ & $-0.07253(18)$ & $4.45(19)$ \\
C14 & $0.34110(16)$ & $0.5985(4)$ & $-0.10433(19)$ & $5.01(20)$ \\
C15 & $0.29672(15)$ & $0.5914(4)$ & $-0.06943(19)$ & $4.65(18)$ \\
C16 & $0.30222(12)$ & $0.5236(3)$ & $-0.00241(17)$ & $3.33(15)$ \\
C21 & $0.29904(11)$ & $0.3906(3)$ & $0.14807(15)$ & $2.41(13)$ \\
C22 & $0.28686(13)$ & $0.5214(4)$ & $0.17752(19)$ & $4.23(17)$ \\
C23 & $0.23688(14)$ & $0.5381(5)$ & $0.20114(19)$ & $5.36(20)$ \\
C24 & $0.19993(13)$ & $0.4238(5)$ & $0.19531(19)$ & $5.10(21)$ \\
C25 & $0.21160(13)$ & $0.2973(4)$ & $0.16518(20)$ & $4.53(18)$ \\
C26 & $0.26063(12)$ & $0.2793(4)$ & $0.14163(17)$ & $3.37(15)$ \\
C31 & $0.36480(11)$ & $0.1804(3)$ & $0.09240(15)$ & $2.43(13)$ \\
C32 & $0.35247(14)$ & $0.1345(4)$ & $0.02114(17)$ & $3.60(16)$ \\
C33 & $0.35229(15)$ & $-0.0116(4)$ & $0.00412(19)$ & $4.43(18)$ \\
C34 & $0.36608(15)$ & $-0.1119(4)$ & $0.05822(21)$ & $4.73(19)$ \\
C35 & $0.37800(16)$ & $-0.0677(4)$ & $0.12935(20)$ & $5.10(20)$ \\
C36 & $0.37776(14)$ & $0.0771(4)$ & $0.14697(17)$ & $3.93(17)$
\end{tabular}

(b) Compound 2

Ru1 0.37036(4)

$\mathrm{Ru} 2 \quad 0.53481(4)$

P1 0.25134(15)

P2 $0.70566(15)$

$01 \quad 0.4633(4)$

$\mathrm{O} 2 \quad 0.5937(4)$

O3 $0.4649(4)$

$04 \quad 0.6025(4)$

O5 $\quad 0.2504(5)$

$06 \quad 0.2354(5)$

$07 \quad 0.4362(5)$

O8 $0.4407(5)$

C1 $0.5532(6)$

C11 0.6112(6)

C12 0.5829(7)

C13 0.6394(8)

C14 0.7236(8)

C15 0.7570(7)

C16 0.6990(6)

C2 $0.5549(6)$

$\mathrm{C} 21 \quad 0.6079(6)$

$\mathrm{C} 22 \quad 0.5731(7)$

C23 0.6207(8)

$\mathrm{C} 24 \quad 0.6969(8)$

C25 $0.7310(7)$

C26 0.6864(7)

C5 $0.2953(6)$

C6 $0.2922(7)$

C7 $0.4749(7)$

C8 $0.4764(7)$

C11A 0.1548(6)

\begin{tabular}{|c|c|c|}
\hline $0.50346(4)$ & $0.050587(24)$ & $2.212(21)$ \\
\hline $0.49549(4)$ & $-0.035473(24)$ & $2.170(21)$ \\
\hline $0.51109(13)$ & $0.14271(8)$ & $2.42(8)$ \\
\hline $0.48653(12)$ & $-0.08990(9)$ & $2.45(8)$ \\
\hline $0.5873(3)$ & $0.09294(23)$ & $3.21(25)$ \\
\hline $0.5798(3)$ & $0.02256(23)$ & $3.14(24)$ \\
\hline $0.4279(3)$ & $0.10344(22)$ & $3.15(25)$ \\
\hline $0.4228(3)$ & $0.03414(23)$ & $3.3(3)$ \\
\hline $0.3848(3)$ & $-0.0161(3)$ & $5.3(3)$ \\
\hline $0.6064(4)$ & $-0.0250(3)$ & $6.1(4)$ \\
\hline $0.6051(3)$ & $-0.1243(3)$ & $5.1(3)$ \\
\hline $0.3781(3)$ & $-0.1173(3)$ & $5.8(4)$ \\
\hline $0.6066(4)$ & $0.0715(3)$ & $2.6(3)$ \\
\hline $0.6654(4)$ & $0.1077(3)$ & $3.0(4)$ \\
\hline $0.6837(5)$ & $0.1690(4)$ & $4.8(5)$ \\
\hline $0.7359(5)$ & $0.2031(5)$ & $6.1(6)$ \\
\hline $0.7678(5)$ & $0.1743(5)$ & $6.7(6)$ \\
\hline $0.7509(5)$ & $0.1135(5)$ & $5.2(5)$ \\
\hline $0.6981(4)$ & $0.0797(4)$ & $3.6(4)$ \\
\hline $0.4040(4)$ & $0.0853(3)$ & $2.4(3)$ \\
\hline $0.3479(4)$ & $0.1261(4)$ & $2.8(3)$ \\
\hline $0.3360(5)$ & $0.1882(4)$ & $4.2(4)$ \\
\hline $0.2816(5)$ & $0.2252(4)$ & $5.4(5)$ \\
\hline $0.2376(5)$ & $0.1994(4)$ & $5.6(5)$ \\
\hline $0.2481(5)$ & $0.1379(4)$ & $4.5(5)$ \\
\hline $0.3043(4)$ & $0.1016(4)$ & $3.5(4)$ \\
\hline $0.4295(4)$ & $0.0096(4)$ & $3.1(4)$ \\
\hline $0.5686(4)$ & $0.0041(4)$ & $3.5(4)$ \\
\hline $0.5628(4)$ & $-0.0903(4)$ & $3.3(4)$ \\
\hline $0.4228(4)$ & $-0.0851(4)$ & $3.5(4)$ \\
\hline $0.4364(4)$ & $0.1410(3)$ & $2.6(3)$ \\
\hline
\end{tabular}

TABLE 2 (continued)

\begin{tabular}{|c|c|c|c|c|}
\hline & $x$ & $y$ & $z$ & $B_{\text {iso }}$ \\
\hline C12A & $0.1621(8)$ & $0.3774(5)$ & $0.1799(4)$ & $5.3(5)$ \\
\hline C13A & $0.0902(10)$ & $0.3190(5)$ & $0.1729(6)$ & $8.1(7)$ \\
\hline C14A & $0.0147(8)$ & $0.3199(5)$ & $0.1269(5)$ & $6.8(6)$ \\
\hline $\mathrm{C} 15 \mathrm{~A}$ & $0.0069(8)$ & $0.3790(6)$ & $0.0879(5)$ & $6.3(6)$ \\
\hline C16A & $0.0751(7)$ & $0.4389(5)$ & $0.0939(4)$ & $4.3(4)$ \\
\hline C11B & $0.3073(5)$ & $0.5065(6)$ & $0.2239(3)$ & 3.3(4) \\
\hline C12B & $0.2433(6)$ & $0.5070(9)$ & $0.2772(3)$ & $5.4(5)$ \\
\hline C13B & $0.2846(6)$ & $0.5041(10)$ & $0.3377(3)$ & $6.6(6)$ \\
\hline C14B & $0.3907(6)$ & $0.5000 \times(9)$ & $0.3459(3)$ & $7.1(6)$ \\
\hline C15B & $0.4562(6)$ & $0.5027(10)$ & $0.2945(3)$ & $7.5(6)$ \\
\hline $\mathrm{C} 16 \mathrm{~B}$ & $0.4151(6)$ & $0.5070(8)$ & $0.2324(3)$ & $5.5(5)$ \\
\hline $\mathrm{C} 11 \mathrm{C}$ & $0.1718(6)$ & $0.5954(4)$ & $0.1490(3)$ & $2.6(3)$ \\
\hline $\mathrm{C} 12 \mathrm{C}$ & $0.0725(7)$ & $0.5962(4)$ & $0.1770(4)$ & $3.5(4)$ \\
\hline $\mathrm{C} 13 \mathrm{C}$ & $0.0197(7)$ & $0.6634(5)$ & $0.1833(4)$ & $5.1(5)$ \\
\hline $\mathrm{C} 14 \mathrm{C}$ & $0.0670(8)$ & $0.7271(5)$ & $0.1631(4)$ & $5.3(5)$ \\
\hline $\mathrm{C} 15 \mathrm{C}$ & $0.1649(8)$ & $0.7270(4)$ & $0.1350(5)$ & $5.1(5)$ \\
\hline $\mathrm{C} 16 \mathrm{C}$ & $0.2179(7)$ & $0.6614(4)$ & $0.1291(4)$ & $3.8(4)$ \\
\hline $\mathrm{C} 21 \mathrm{~A}$ & $0.7734(6)$ & $0.3988(4)$ & $-0.0822(4)$ & $3.1(4)$ \\
\hline C22A & $0.8811(8)$ & $0.3940(5)$ & $-0.0858(5)$ & $5.7(5)$ \\
\hline C23A & $0.9326(8)$ & $0.3252(6)$ & $-0.0836(5)$ & $6.9(6)$ \\
\hline $\mathrm{C} 24 \mathrm{~A}$ & $0.8759(9)$ & $0.2631(5)$ & $-0.0759(5)$ & $6.4(6)$ \\
\hline C25A & $0.7688(8)$ & $0.2669(5)$ & $-0.0734(4)$ & $5.1(5)$ \\
\hline $\mathrm{C} 26 \mathrm{~A}$ & $0.7187(7)$ & $0.3342(5)$ & $-0.0758(4)$ & $4.2(4)$ \\
\hline C21B & $0.6996(5)$ & $0.4994(6)$ & $-0.1770(3)$ & $2.9(3)$ \\
\hline C22B & $0.6624(7)$ & $0.5667(4)$ & $-0.2000(4)$ & $3.7(4)$ \\
\hline C23B & $0.6575(7)$ & $0.5795(5)$ & $-0.2653(4)$ & $4.7(5)$ \\
\hline C24B & $0.6886(7)$ & $0.5263(5)$ & $-0.3075(4)$ & $5.0(5)$ \\
\hline C25B & $0.7266(8)$ & $0.4600(5)$ & $-0.2864(4)$ & $5.8(6)$ \\
\hline C26B & $0.7315(7)$ & $0.4468(5)$ & $-0.2202(4)$ & 4.1(4) \\
\hline $\mathrm{C} 21 \mathrm{C}$ & $0.8055(6)$ & $0.5508(4)$ & $-0.0613(3)$ & $2.7(3)$ \\
\hline $\mathrm{C} 22 \mathrm{C}$ & $0.8303(7)$ & $0.5474(5)$ & $0.0023(4)$ & $4.4(5)$ \\
\hline $\mathrm{C} 23 \mathrm{C}$ & $0.9085(8)$ & $0.5927(6)$ & $0.0272(4)$ & $6.2(6)$ \\
\hline $\mathrm{C} 24 \mathrm{C}$ & $0.9616(8)$ & $0.6414(6)$ & $-0.0103(5)$ & $6.9(6)$ \\
\hline $\mathrm{C} 25 \mathrm{C}$ & $0.9362(8)$ & $0.6466(6)$ & $-0.0720(5)$ & $6.6(6)$ \\
\hline $\mathrm{C} 26 \mathrm{C}$ & $0.8616(8)$ & $0.6020(5)$ & $-0.0981(4)$ & $5.2(5)$ \\
\hline
\end{tabular}

(c) Compound 3

$\begin{array}{lll}\text { Ru1 } & 0.38673(5) & 0.27446(5) \\ \text { Ru2 } & 0.29736(5) & 0.16748(5) \\ \text { P1 } & 0.42980(17) & 0.37113(15) \\ \text { P2 } & 0.17462(16) & 0.09318(15) \\ \text { O1 } & 0.2423(4) & 0.3901(4) \\ \text { O2 } & 0.1984(4) & 0.3189(4) \\ \text { O3 } & 0.2765(4) & 0.2095(4) \\ \text { O4 } & 0.1769(4) & 0.1587(4) \\ \text { C1 } & 0.1891(6) & 0.3876(5) \\ \text { C2 } & 0.0935(8) & 0.4855(7) \\ \text { F1 }{ }^{b} & 0.1356(7) & 0.5597(6) \\ \text { F2 }{ }^{b} & 0.0682(7) & 0.5387(6) \\ \text { F3 }{ }^{b} & -0.0017(7) & 0.4602(7) \\ \text { F4 } & 0.0584(8) & 0.4861(7) \\ \text { C3 } & 0.1974(7) & 0.1769(6) \\ \text { C4 } & 0.1032(10) & 0.1576(10) \\ \text { F5 }{ }^{b} & 0.1579(9) & 0.0977(8) \\ \text { F6 }{ }^{b} & 0.0869(7) & 0.2219(7) \\ \text { F7 }{ }^{b} & 0.0074(8) & 0.1734(7) \\ \text { F8 }{ }^{b} & 0.0916(9) & 0.0596(8) \\ \text { C5 } & 0.5075(6) & 0.1691(6) \\ \text { O5 } & 0.5829(5) & 0.1028(4) \\ \text { C6 } & 0.4657(7) & 0.3362(6) \\ \text { O6 }^{b} & 0.5117(5) & 0.3760(5) \\ \text { C7 } & 0.4101(7) & 0.1769(6)\end{array}$

$\begin{array}{lc}0.23389(5) & 2.57(3) \\ 0.14176(5) & 2.53(3) \\ 0.34295(15) & 2.74(10) \\ 0.08186(16) & 2.69(9) \\ 0.2154(4) & 3.9(3) \\ 0.1026(4) & 3.3(3) \\ 0.3712(4) & 3.6(3) \\ 0.2873(4) & 3.5(3) \\ 0.1534(6) & 3.1(4) \\ 0.1453(9) & 7.5(8) \\ 0.0778(6) & 8.46(23) \\ 0.2141(6) & 7.78(21) \\ 0.1729(7) & 9.3(3) \\ 0.0615(7) & 10.9(3) \\ 0.3634(6) & 3.5(4) \\ 0.4671(8) & 8.6(8) \\ 0.5353(8) & 12.3(3) \\ 0.5229(7) & 8.82(24) \\ 0.4530(7) & 9.7(3) \\ 0.4792(8) & 11.6(3) \\ 0.2409(6) & 3.4(4) \\ 0.2437(5) & 5.3(4) \\ 0.1108(6) & 3.6(4) \\ 0.0335(4) & 5.7(4) \\ 0.0227(6) & 3.5(4)\end{array}$


TABLE 2 (continued)

\begin{tabular}{|c|c|c|c|c|}
\hline & $x$ & $y$ & $z$ & $B_{\text {iso }}$ \\
\hline O7 & $0.4844(5)$ & $0.1763(5)$ & $-0.0460(4)$ & $5.6(4)$ \\
\hline $\mathrm{C} 8$ & $0.3832(6)$ & $0.0431(6)$ & $0.1831(6)$ & $3.2(4)$ \\
\hline 08 & $0.4376(5)$ & $-0.0339(4)$ & $0.2088(5)$ & $4.9(3)$ \\
\hline C11A & $0.5145(6)$ & $0.2882(6)$ & $0.4287(5)$ & $2.9(4)$ \\
\hline C12A & $0.6011(7)$ & $0.3199(6)$ & $0.4469(6)$ & $3.8(4)$ \\
\hline $\mathrm{C} 13 \mathrm{~A}$ & $0.6669(7)$ & $0.2539(8)$ & $0.5087(7)$ & $5.2(6)$ \\
\hline C14A & $0.6444(8)$ & $0.1565(8)$ & $0.5530(7)$ & $5.7(6)$ \\
\hline C15A & $0.5566(8)$ & $0.1238(6)$ & $0.5372(7)$ & $5.0(5)$ \\
\hline C16A & $0.4914(7)$ & $0.1903(6)$ & $0.4751(6)$ & $3.9(5)$ \\
\hline C11B & $0.5146(6)$ & $0.4699(6)$ & $0.2757(6)$ & $2.9(4)$ \\
\hline $\mathrm{C} 12 \mathrm{~B}$ & $0.4865(7)$ & $0.5707(6)$ & $0.2942(6)$ & $4.0(5)$ \\
\hline C13B & $0.5578(8)$ & $0.6407(7)$ & $0.2420(7)$ & $5.4(6)$ \\
\hline C14B & $0.6548(8)$ & $0.6101(7)$ & $0.1743(7)$ & $5.8(6)$ \\
\hline $\mathrm{C} 15 \mathrm{~B}$ & $0.6833(7)$ & $0.5107(8)$ & $0.1536(7)$ & $5.3(5)$ \\
\hline C16B & $0.6136(7)$ & $0.4401(6)$ & $0.2036(6)$ & $4.1(4)$ \\
\hline $\mathrm{C} 11 \mathrm{C}$ & $0.3111(6)$ & $0.4397(5)$ & $0.4274(6)$ & $3.0(4)$ \\
\hline $\mathrm{C} 12 \mathrm{C}$ & $0.2275(7)$ & $0.5071(6)$ & $0.3871(6)$ & $4.2(5)$ \\
\hline $\mathrm{C} 13 \mathrm{C}$ & $0.1376(7)$ & $0.5604(7)$ & $0.4492(7)$ & $5.1(5)$ \\
\hline $\mathrm{C} 14 \mathrm{C}$ & $0.1274(8)$ & $0.5477(7)$ & $0.5513(7)$ & $5.6(5)$ \\
\hline $\mathrm{C} 15 \mathrm{C}$ & $0.2080(8)$ & $0.4802(7)$ & $0.5941(7)$ & $5.4(6)$ \\
\hline $\mathrm{C} 16 \mathrm{C}$ & $0.3004(7)$ & $0.4270(6)$ & $0.5328(6)$ & 4.1(4) \\
\hline $\mathrm{C} 21 \mathrm{~A}$ & $0.1758(6)$ & $0.1384(5)$ & $-0.0532(6)$ & $3.0(4)$ \\
\hline $\mathrm{C} 22 \mathrm{~A}$ & $0.2772(7)$ & $0.1210(6)$ & $-0.1255(6)$ & $3.6(4)$ \\
\hline $\mathrm{C} 23 \mathrm{~A}$ & $0.2836(8)$ & $0.1560(7)$ & $-0.2288(6)$ & $5.0(5)$ \\
\hline C24A & $0.1883(9)$ & $0.2086(7)$ & $-0.2604(7)$ & $5.7(6)$ \\
\hline $\mathrm{C} 25 \mathrm{~A}$ & $0.0890(9)$ & $0.2258(7)$ & $-0.1899(7)$ & $6.0(6)$ \\
\hline $\mathrm{C} 26 \mathrm{~A}$ & $0.0815(7)$ & $0.1905(6)$ & $-0.0867(6)$ & $4.0(5)$ \\
\hline C21B & $0.0282(6)$ & $0.1193(6)$ & $0.1506(6)$ & $3.0(4)$ \\
\hline $\mathrm{C} 22 \mathrm{~B}$ & $-0.0363(7)$ & $0.0430(6)$ & $0.1867(7)$ & $4.2(5)$ \\
\hline C23B & $-0.1451(7)$ & $0.0658(7)$ & $0.2409(7)$ & $5.1(5)$ \\
\hline C24B & $-0.1919(7)$ & $0.1627(8)$ & $0.2641(7)$ & $5.3(5)$ \\
\hline $\mathrm{C} 25 \mathrm{~B}$ & $-0.1295(7)$ & $0.2395(7)$ & $0.2274(9)$ & $6.7(7)$ \\
\hline $\mathrm{C} 26 \mathrm{~B}$ & $-0.0194(7)$ & $0.2180(7)$ & $0.1721(8)$ & $5.5(6)$ \\
\hline $\mathrm{C} 21 \mathrm{C}$ & $0.2070(6)$ & $-0.0487(5)$ & $0.0972(6)$ & $3.0(4)$ \\
\hline $\mathrm{C} 22 \mathrm{C}$ & $0.2173(7)$ & $-0.1043(6)$ & $0.1930(7)$ & $4.4(5)$ \\
\hline $\mathrm{C} 23 \mathrm{C}$ & $0.2405(8)$ & $-0.2119(7)$ & $0.2092(7)$ & $5.4(6)$ \\
\hline $\mathrm{C} 24 \mathrm{C}$ & $0.2549(8)$ & $-0.2636(7)$ & $0.1319(8)$ & $5.9(6)$ \\
\hline $\mathrm{C} 25 \mathrm{C}$ & $0.2440(8)$ & $-0.2110(7)$ & $0.0360(7)$ & $5.2(5)$ \\
\hline $\mathrm{C} 26 \mathrm{C}$ & $0.2192(7)$ & $-0.1021(6)$ & $0.0192(6)$ & $4.0(4)$ \\
\hline
\end{tabular}

${ }^{a} B_{\text {iso }}$ is the mean of the principal axes of the thermal ellipsoid; b occupancy $=0.75$.

lengths, we compare Mo- $\mathrm{O}$ (equatorial, bridging carboxylates), Mo-CO (equatorial carbonyl) and Mo-L (axial ligands) distances from all the reported structures of $\left[\mathrm{Ru}_{2}\left(\mathrm{O}_{2} \mathrm{CR}\right)_{2}(\mathrm{CO})_{4} \mathrm{~L}_{2}\right]\left(\mathrm{L}=\mathrm{CO} . \mathrm{PPh}_{3}, \mathrm{PBu}_{3}\right.$, py, $\mathrm{H}_{2} \mathrm{O}$, or $\mathrm{PhCO}_{2} \mathrm{H} ; \mathrm{R}=\mathrm{Me}, \mathrm{CF}_{3},{ }^{n} \mathrm{Pr}$, or $4-\mathrm{FC}_{6} \mathrm{H}_{4}$ ) and $\left[\mathrm{Ru}_{2}\left(\mathrm{O}_{2} \mathrm{CPh}\right)_{2}(\mathrm{CO})_{4}\right]_{\infty}[10,11,15,16]$ in Table 4 . From this table, the mild inductive effect of $R$ is reflected in the bond lengths of $2.736 \AA$ for $1(R=M e$, $\left.\mathrm{L}=\mathrm{PPh}_{3}\right) ; 2.741 \AA$ for $2\left(\mathrm{R}=\mathrm{Ph}, \mathrm{L}=\mathrm{PPh}_{3}\right)$ and 2.728 $\AA$ for $3\left(R=C F_{3}, L=P_{3}\right)$ but a rather strong axial coordination effect is shown in terms of $R, L$, and $\mathrm{d}(\mathrm{Ru}-\mathrm{Ru})$ : Me, CO, 2.689; $\mathrm{Me}$, py, 2.678; $\mathrm{Me}, \mathrm{PPh}_{3}$, 2.736; Ph, CO, 2.704; $\mathrm{Ph}, \mathrm{PhCO}_{2} \mathrm{H}, 2.637 ; \mathrm{Ph}, \mathrm{PPh}_{3}$, $2.741 \AA$. The differing sensitivities of $\mathrm{Ru}-\mathrm{Ru}$ lengths to axial coordination and inductive effect can be ratio-

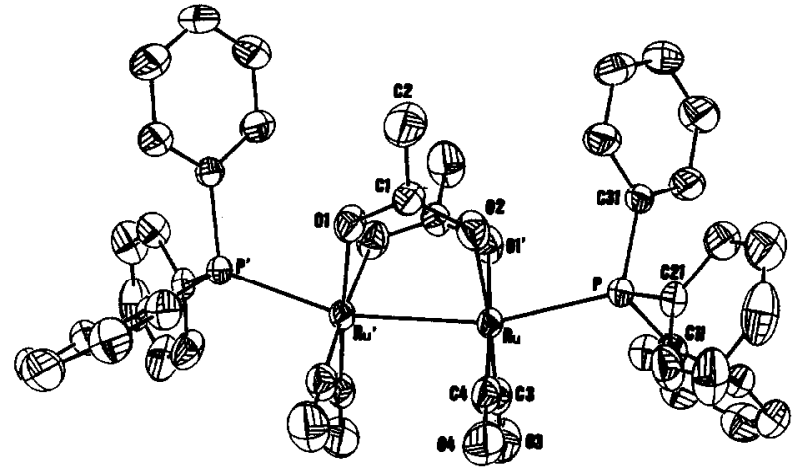

Fig. 1. ORTEP drawing of $\left[\mathrm{Ru}_{2}\left(\mathrm{O}_{2} \mathrm{CMe}\right)_{2}(\mathrm{CO})_{4}\left(\mathrm{PPh}_{3}\right)_{2}\right]$ (1) with the numbering scheme. $A \mathrm{C}_{2}$ axis is imposed crystallographically at the centre of the $\mathrm{Ru}-\mathrm{Ru}$ bond. Thermal ellipsoids are drawn at the $50 \%$ probability level.

nalized by applying the molecular-orbital calculation results for HVCs [12c,g, 21-24] and the formal electron configuration of $\sigma^{2} \pi^{4} \delta^{2} \delta^{* 2} \pi^{* 4}$ in the $\mathrm{Ru}_{2}$ compounds in Table 4 . When $\pi$-acids such as CO coordinate axially with the $R u_{2}$ dimer, electron-drainage from the filled $\mathrm{M}-\mathrm{M}$ antibonding orbitals ( $\delta^{*}$ or $\pi^{*}$ ) to the $\mathrm{CO} \pi^{*}$ orbitals strengthens the $\mathrm{M}-\mathrm{M}$ bonding interaction by increasing the $\mathbf{M}-\mathbf{M}$ bond order. However, when the axial ligands are strong $\sigma$-donors with or without some weak $\pi$-accepting ability such as py or $\mathrm{PPh}_{3}$, the $\mathbf{M}-\mathbf{M}$ bond order decreases by bonding interactions through the empty $\mathbf{M}-\mathbf{M}$ antibonding orbitals $\left(\sigma^{*}\right)$. Following the results of calculation, it is obvious that the through-bond coupling [21,25], which is negative for 1,2 , or 3 , is determined primarily by the relative disposition of the bridging carboxylates with respect to the metal centers (i.e., the $\mathrm{Ru}-\mathrm{O}$ distances). As evidenced in the averaged $R u-O$ and $R u-R u$ dis-

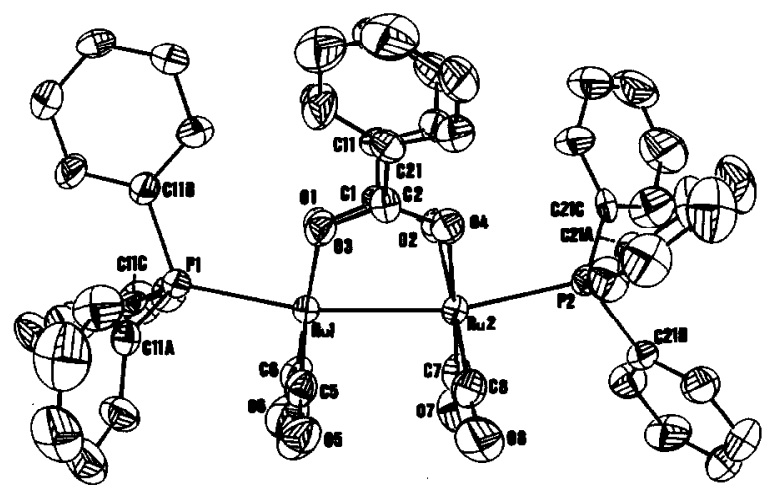

Fig. 2. ORTEP drawing of $\left[\mathrm{Ru}_{2}\left(\mathrm{O}_{2} \mathrm{CPh}\right)_{2}(\mathrm{CO})_{4}\left(\mathrm{PPh}_{3}\right)_{2}\right]$ (2) with the numbering scheme. One disordered $\mathrm{CF}_{3}$ group presented by one carbon atom and four fluorine atoms with occupancy $=0.75$ is drawn whereas the other disordered $\mathrm{CF}_{3}$ group is not drawn for clarity. Atom $C(5)$ hides behind atoms $C(6)$ and $O(6)$. Thermal ellipsoids are drawn at the $50 \%$ probability level. 
TABLE 3. Selected bond lengths and angles for 1-3

\begin{tabular}{|c|c|c|c|}
\hline \multicolumn{4}{|l|}{ (a) Compound 1} \\
\hline $\mathbf{R u}-\mathbf{R} \mathbf{u}^{\prime}$ & $2.7360(9)$ & $P-C(21)$ & $1.826(3)$ \\
\hline $\mathbf{R u}-\mathbf{P}$ & $2.4508(10)$ & $P-C(31)$ & $1.825(3)$ \\
\hline $\mathbf{R u}-\mathbf{O}(1)$ & $2.1089(20)$ & $O(1)-C(1)$ & $1.233(3)$ \\
\hline $\mathbf{R u}-\mathbf{O}(2)$ & $2.1289(21)$ & $O(2)-C(1)$ & $1.289(4)$ \\
\hline $\mathrm{Ru}-\mathrm{C}(3)$ & $1.831(3)$ & $\mathrm{C}(1)-\mathrm{C}(2)$ & $1.494(5)$ \\
\hline $\mathrm{Ru}-\mathrm{C}(4)$ & $1.836(3)$ & $C(3)-O(3)$ & $1.150(4)$ \\
\hline $\mathrm{P}-\mathrm{C}(11)$ & $1.821(3)$ & $C(4)-O(4)$ & $1.150(4)$ \\
\hline $\mathbf{R} \mathbf{u}^{\prime}-\mathbf{R u}-\mathbf{P}$ & $163.539(23)$ & $C(3)-R u-C(4)$ & $88.34(14)$ \\
\hline $\mathbf{R u} \mathbf{u}^{\prime}-\mathbf{R u}-\mathbf{O}(1)^{\prime}$ & $83.05(5)$ & $\mathrm{Ru}-\mathrm{P}-\mathrm{C}(11)$ & $114.50(10)$ \\
\hline $\mathbf{R u}-\mathbf{R u}-\mathrm{O}(2)$ & $83.25(6)$ & $\mathrm{Ru}-\mathrm{P}-\mathrm{C}(21)$ & $117.52(10)$ \\
\hline $\mathbf{R} \mathbf{u}^{\prime}-\mathbf{R u}-\mathbf{C}(3)$ & $92.63(9)$ & $\mathrm{Ru}-\mathrm{P}-\mathrm{C}(31)$ & $113.32(9)$ \\
\hline $\mathbf{R u}-\mathbf{R u}-\mathrm{C}(4)$ & $94.41(9)$ & $C(11)-P-C(21)$ & $103.68(13)$ \\
\hline$P-R u-O(1)$ & $82.98(6)$ & $\mathrm{C}(11)-\mathrm{P}-\mathrm{C}(31)$ & $102.97(13)$ \\
\hline P-Ru-O(2) & $87.13(6)$ & $\mathrm{C}(21)-\mathrm{P}-\mathrm{C}(31)$ & $103.13(13)$ \\
\hline P-Ru-C(3) & $96.53(9)$ & $\mathrm{Ru}-\mathrm{O}(1)^{\prime}-\mathrm{C}(1)^{\prime}$ & $125.80(19)$ \\
\hline P-Ru-C(4) & $99.48(9)$ & $R u-O(2)-C(1)$ & $123.12(18)$ \\
\hline$O(1)^{\prime}-\mathrm{Ru}-\mathrm{O}(2)$ & $85.94(9)$ & $O(1)-C(1)-O(2)$ & $124.4(3)$ \\
\hline$O(1)^{\prime}-R u-C(3)$ & $92.09(12)$ & $\mathrm{O}(1)-\mathrm{C}(1)-\mathrm{C}(2)$ & $118.3(3)$ \\
\hline$O(1)^{\prime}-\mathrm{Ru}-\mathrm{C}(4)$ & $177.44(10)$ & $O(2)-C(1)-C(2)$ & $117.3(3)$ \\
\hline$O(2)-R u-C(3)$ & $175.61(10)$ & $\mathrm{Ru}-\mathrm{C}(3)-\mathrm{O}(3)$ & $179.4(3)$ \\
\hline$O(2)-R u-C(4)$ & $93.46(12)$ & $\mathrm{Ru}-\mathrm{C}(4)-\mathrm{O}(4)$ & $178.3(3)$ \\
\hline \multicolumn{4}{|l|}{ (b) Compound 2} \\
\hline $\mathrm{Ru}(1)-\mathbf{R u}(2)$ & $2.7411(8)$ & $P(2)-C(21 A)$ & $1.817(8)$ \\
\hline $\mathbf{R u}(1)-\mathbf{P}(1)$ & $2.4367(19)$ & $P(2)-C(21 B)$ & $1.828(6)$ \\
\hline $\mathrm{Ru}(1)-\mathrm{O}(1)$ & $2.114(5)$ & $P(2)-C(21 C)$ & $1.816(7)$ \\
\hline $\mathrm{Ru}(1)-\mathrm{O}(3)$ & $2.124(5)$ & $O(1)-C(1)$ & $1.266(9)$ \\
\hline $\mathrm{Ru}(1)-\mathrm{C}(5)$ & $1.851(8)$ & $O(2)-C(1)$ & $1.239(9)$ \\
\hline $\mathrm{Ru}(1)-\mathrm{C}(6)$ & $1.819(8)$ & $O(3)-C(2)$ & $1.271(9)$ \\
\hline $\mathrm{Ru}(2)-\mathrm{P}(2)$ & $2.4364(20)$ & $O(4)-C(2)$ & $1.269(8)$ \\
\hline $\operatorname{Ru}(2)-O(2)$ & $2.088(5)$ & $O(5)-C(5)$ & $1.127(9)$ \\
\hline $\mathrm{Ru}(2)-\mathrm{O}(4)$ & $2.138(5)$ & $O(6)-C(6)$ & $1.162(10)$ \\
\hline $\operatorname{Ru}(2)-C(7)$ & $1.835(8)$ & $O(7)-C(7)$ & $1.154(9)$ \\
\hline $\mathrm{Ru}(2)-\mathrm{C}(8)$ & $1.830(8)$ & $O(8)-C(8)$ & $1.146(9)$ \\
\hline $\mathrm{Ru}(2)-\mathrm{C}(8)$ & $1.830(8)$ & $O(8)-C(8)$ & $1.146(9)$ \\
\hline$P(1)-C(11 A)$ & $1.824(7)$ & $C(1)-C(11)$ & $1.497(10)$ \\
\hline$P(1)-C(11 B)$ & $1.832(6)$ & $C(2)-C(21)$ & $1.486(10)$ \\
\hline$P(1)-C(11 C)$ & $1.835(7)$ & & \\
\hline $\mathbf{R u}(2)-\mathbf{R u}(1)-\mathbf{P}(1)$ & $168.89(5)$ & $C(5)-R u(1)-C(6)$ & 87.2(4) \\
\hline$R u(2)-R u(1)-O(1)$ & $83.83(13)$ & $\mathrm{Ru}(1)-\mathrm{Ru}(2)-\mathrm{P}(2)$ & $166.91(5)$ \\
\hline $\mathrm{Ru}(2)-\mathrm{Ru}(1)-\mathrm{O}(3)$ & $83.14(13)$ & $\mathrm{Ru}(1)-\mathrm{Ru}(2)-O(2)$ & $81.46(13)$ \\
\hline$R u(2)-R u(1)-C(5)$ & $92.67(24)$ & $R u(1)-R u(2)-O(4)$ & $83.75(14)$ \\
\hline$R u(2)-R u(1)-C(6)$ & $95.50(25)$ & $R u(1)-R u(2)-C(7)$ & $93.45(24)$ \\
\hline $\mathrm{P}(1)-\mathrm{Ru}(1)-\mathrm{O}(1)$ & $88.36(14)$ & $R u(1)-R u(2)-C(8)$ & $95.9(3)$ \\
\hline$P(1)-R u(1)-O(3)$ & $88.53(14)$ & $P(2)-R u(2)-O(2)$ & $90.26(14)$ \\
\hline$P(1)-R u(1)-C(5)$ & $95.13(24)$ & $P(2)-R u(2)-O(4)$ & $85.51(14)$ \\
\hline$P(1)-R u(1)-C(6)$ & $92.78(25)$ & $P(2)-R u(2)-G(7)$ & $96.81(25)$ \\
\hline$O(1)-R u(1)-O(3)$ & $86.59(20)$ & $P(2)-R u(2)-C(8)$ & $92.5(3)$ \\
\hline$O(1)-R u(1)-C(5)$ & $176.5(3)$ & $O(2)-R u(2)-O(4)$ & $85.48(20)$ \\
\hline$O(1)-R u(1)-C(6)$ & $93.0(3)$ & $O(2)-R u(2)-C(7)$ & $90.9(3)$ \\
\hline$O(3)-R u(1)-C(5)$ & 93.1(3) & $O(2)-R u(2)-C(8)$ & $177.1(3)$ \\
\hline$O(3)-R u(1)-C(6)$ & $178.6(3)$ & $\mathrm{O}(4)-\mathrm{Ru}(2)-\mathrm{O}(7)$ & $175.7(3)$ \\
\hline$R u(1)-C(5)-O(5)$ & $179.0(7)$ & $O(4)-R u(2)-C(8)$ & $95.5(3)$ \\
\hline$R u(1)-C(6)-O(6)$ & $174.6(8)$ & $C(7)-R u(2)-C(8)$ & $88.1(3)$ \\
\hline $\mathrm{Ru}(2)-\mathrm{C}(7)-\mathrm{O}(7)$ & $179.2(7)$ & $O(1)-C(1)-O(2)$ & $123.2(7)$ \\
\hline $\mathrm{Ru}(2)-\mathrm{C}(8)-\mathrm{O}(8)$ & $178.5(7)$ & $O(1)-C(1)-C(11)$ & $117.2(6)$ \\
\hline $\mathrm{Ru}(1)-\mathrm{O}(1)-\mathrm{C}(1)$ & $123.3(4)$ & $O(2)-C(1)-C(11)$ & $119.6(7)$ \\
\hline $\mathrm{Ru}(2)-\mathrm{O}(2)-\mathrm{C}(1)$ & $128.2(5)$ & $O(3)-C(2)-O(4)$ & $125.3(6)$ \\
\hline$R u(1)-O(3)-C(2)$ & $124.6(4)$ & $O(3)-C(2)-C(21)$ & $117.8(6)$ \\
\hline$R u(2)-O(4)-C(2)$ & $123.2(5)$ & $O(4)-C(2)-C(21)$ & $116.9(6)$ \\
\hline
\end{tabular}


TABLE 3 (continued)

\begin{tabular}{|c|c|c|c|c|}
\hline \multicolumn{5}{|l|}{ (c) Compound 3} \\
\hline $\mathbf{R u}(1)-\mathbf{R u}(2)$ & $2.7276(9)$ & $P(1)-C(11 A)$ & $1.832(8)$ & \\
\hline $\mathrm{Ru}(1)-\mathrm{P}(1)$ & $2.4430(21)$ & $P(1)-C(11 B)$ & $1.828(7)$ & \\
\hline $\mathrm{Ru}(1)-\mathrm{O}(1)$ & $2.138(5)$ & $P(1)-C(11 C)$ & $1.816(7)$ & \\
\hline $\mathrm{Ru}(1)-O(3)$ & $2.151(5)$ & $P(2)-C(21 A)$ & $1.820(8)$ & \\
\hline $\mathrm{Ru}(1)-\mathrm{C}(5)$ & $1.831(8)$ & $P(2)-C(21 B)$ & $1.821(7)$ & \\
\hline $\mathrm{Ru}(1)-\mathrm{C}(6)$ & $1.833(8)$ & $P(2)-C(21 C)$ & $1.828(7)$ & \\
\hline $\mathrm{Ru}(2)-\mathrm{P}(2)$ & $2.4492(21)$ & $O(1)-C(1)$ & $1.245(9)$ & \\
\hline$R u(2)-O(2)$ & $2.162(5)$ & $O(2)-C(1)$ & $1.248(9)$ & \\
\hline $\mathrm{Ru}(2)-\mathrm{O}(4)$ & $2.146(5)$ & $O(3)-C(3)$ & $1.231(9)$ & \\
\hline $\mathrm{Ru}(2)-\mathrm{C}(7)$ & $1.847(8)$ & $O(4)-C(3)$ & $1.239(9)$ & \\
\hline \multirow[t]{2}{*}{$\mathrm{Ru}(2)-\mathrm{C}(8)$} & $1.832(8)$ & $C(1)-C(2)$ & $1.569(11)$ & \\
\hline & & $C(3)-C(4)$ & $1.596(13)$ & \\
\hline \multirow[t]{6}{*}{$R u(2)-C(8)$} & $1.832(8)$ & $C(1)-C(2)$ & $1.569(11)$ & \\
\hline & & $C(3)-C(4)$ & $1.596(13)$ & * \\
\hline & & $C(5)-O(5)$ & $1.144(9)$ & \\
\hline & & $C(6)-O(6)$ & $1.139(10)$ & \\
\hline & & $C(7)-O(7)$ & $1.131(9)$ & \\
\hline & & $C(8)-O(8)$ & $1.142(9)$ & \\
\hline $\mathbf{R u}(2)-\mathbf{R u}(1)-\mathrm{P}(1)$ & $168.11(6)$ & $O(3)-R u(1)-C(6)$ & 173.1(3) & \\
\hline$R u(2)-R u(1)-O(1)$ & $82.36(13)$ & $C(5)-R u(1)-C(6)$ & $89.7(4)$ & \\
\hline$R u(2)-R u(1)-O(3)$ & $83.15(13)$ & $\mathbf{R u}(1)-\mathbf{R u}(2)-\mathbf{P}(2)$ & $166.22(5)$ & \\
\hline$R u(2)-R u(1)-C(5)$ & $92.21(23)$ & $R u(1)-R u(2)-O(2)$ & $83.08(13)$ & \\
\hline$R u(2)-R u(1)-C(6)$ & $92.92(24)$ & $\mathrm{Ru}(1)-\mathrm{Ru}(2)-\mathrm{O}(4)$ & $82.20(13)$ & \\
\hline$P(1)-R u(1)-O(1)$ & $91.27(14)$ & $\mathrm{Ru}(1)-\mathrm{Ru}(2)-\mathrm{C}(7)$ & $92.90(23)$ & \\
\hline$P(1)-R u(1)-O(3)$ & $86.28(14)$ & $\mathrm{Ru}(1)-\mathrm{Ru}(2)-\mathrm{C}(8)$ & $92.69(23)$ & \\
\hline$P(1)-R u(1)-C(5)$ & $94.32(23)$ & $P(2)-R u(2)-O(2)$ & $87.88(14)$ & \\
\hline$P(1)-R u(1)-C(6)$ & $97.04(24)$ & $\mathrm{P}(2)-\mathrm{Ru}(2)-\mathrm{O}(4)$ & $86.77(14)$ & \\
\hline$O(1)-R u(1)-O(3)$ & $84.67(21)$ & $P(2)-R u(2)-C(7)$ & $98.37(24)$ & \\
\hline$O(1)-R u(1)-C(5)$ & $174.4(3)$ & $P(2)-R u(2)-C(8)$ & $95.98(23)$ & \\
\hline$O(1)-R u(1)-C(6)$ & $89.2(3)$ & & & \\
\hline$O(3)-R u(1)-C(5)$ & $96.1(3)$ & & & \\
\hline $\operatorname{Ru}(1)-O(1)-C(1)$ & $121.0(4)$ & $O(2)-R u(2)-O(4)$ & $85.67(19)$ & \\
\hline $\mathrm{Ru}(2)-O(2)-\mathrm{C}(1)$ & $118.6(5)$ & $O(2)-R u(2)-C(7)$ & $95.8(3)$ & \\
\hline $\mathrm{Ru}(1)-O(3)-\mathrm{C}(3)$ & $118.9(5)$ & $O(2)-R u(2)-C(8)$ & $175.5(3)$ & \\
\hline $\mathbf{R u}(2)-O(4)-C(3)$ & $120.6(5)$ & $O(4)-R u(2)-C(7)$ & $174.7(3)$ & \\
\hline$O(1)-C(1)-O(2)$ & $129.8(7)$ & $O(4)-R u(2)-C(8)$ & $92.2(3)$ & \\
\hline$O(1)-C(1)-C(2)$ & $112.1(7)$ & $\mathrm{C}(7)-\mathrm{Ru}(2)-\mathrm{C}(8)$ & $85.9(3)$ & \\
\hline$O(2)-C(1)-C(2)$ & $118.0(7)$ & & & \\
\hline$R u(1)-C(5)-O(5)$ & $178.8(7)$ & & & \\
\hline$R u(1)-C(6)-O(6)$ & $177.9(7)$ & & & \\
\hline $\mathrm{Ru}(2)-\mathrm{C}(7)-\mathrm{O}(7)$ & $174.3(7)$ & & & \\
\hline $\operatorname{Ru}(2)-C(8)-O(8)$ & $179.3(7)$ & & & \\
\hline
\end{tabular}

TABLE 4. Structures of $\left[\mathrm{Ru}_{2}\left(\mathrm{O}_{2} \mathrm{CR}\right)_{2}(\mathrm{CO})_{4} \mathrm{~L}_{2}\right]$

\begin{tabular}{|c|c|c|c|c|c|c|}
\hline \multicolumn{2}{|l|}{ Compound } & \multicolumn{5}{|c|}{ Averaged interatomic distances, $\AA$} \\
\hline$\overline{\mathbf{R}}$ & $2 \mathrm{~L}$ & $\mathrm{Ru}-\mathrm{O}$ & $\mathrm{Ru}-\mathrm{CO}$ & $\mathbf{R u}-\mathbf{L}$ & $\mathbf{R u}-\mathbf{R u}$ & Reference \\
\hline$\overline{\mathrm{Me}}$ & $2 \mathrm{CO}$ & 2.094 & 1.845 & 1.976 & 2.689 & 11 \\
\hline $\mathrm{Me}$ & 2 py & $-a$ & 1.84 & $-a$ & 2.678 & 10 \\
\hline $\mathbf{M e}$ & $2 \mathrm{PPh}_{3}$ & 2.119 & 1.834 & 2.451 & 2.736 & This work \\
\hline $\mathrm{CF}_{3}$ & $2 \mathrm{PPh}_{3}$ & 2.149 & 1.836 & 2.446 & 2.728 & This work \\
\hline $\operatorname{Pr}^{n}$ & $2 \mathrm{P}^{t} \mathrm{Bu}_{3}$ & 2.13 & 1.83 & 2.622 & 2.728 & 15 \\
\hline $4-\mathrm{F}-\mathrm{C}_{4} \mathrm{H}_{4}$ & $\left\{\mathrm{CO}, \mathrm{H}_{2} \mathrm{O}\right\}$ & 2.119 & 1.851 & $1.999^{b}, 2.999^{c}$ & 2.649 & 16 \\
\hline $\mathrm{Ph}$ & $2 \mathrm{PhCO}_{2} \mathrm{H}^{\mathrm{d}}$ & 2.134 & 1.834 & 2.265 & 2.637 & 16 \\
\hline $\mathrm{Ph}$ & $2 \mathrm{PhCO}_{2} \mathrm{e}$ & 2.130 & 1.829 & 2.299 & 2.639 & 11 \\
\hline $\mathrm{Ph}$ & $2 \mathrm{CO}$ & 2.108 & 1.840 & 2.013 & 2.704 & 11 \\
\hline $\mathrm{Ph}$ & $2 \mathrm{PPh}_{3}$ & 2.116 & 1.834 & 2.437 & 2.741 & This work \\
\hline
\end{tabular}

${ }^{\mathrm{a}}$ Only the $\mathrm{Ru}-\mathrm{Ru}$ and $\mathrm{Ru}-\mathrm{CO}$ distances were cited previously [10]; ${ }^{\mathrm{b}} \mathrm{Ru}-\mathrm{CO}$ distance; ${ }^{\mathrm{c}} \mathrm{Ru}-\mathrm{OH}_{2}$ distance; ${ }^{\mathrm{d}}$ There exist two intramolecular hydrogen bonds between $\mathrm{PhCO}_{2} \mathrm{H}$ and one bridging $\mathrm{PhCOO}$ ligand; ${ }^{\mathrm{c}}$ Oxygen atoms of the neighboring bridging carboxylates coordinate with the $\mathrm{Ru}$ atoms in axial positions. 


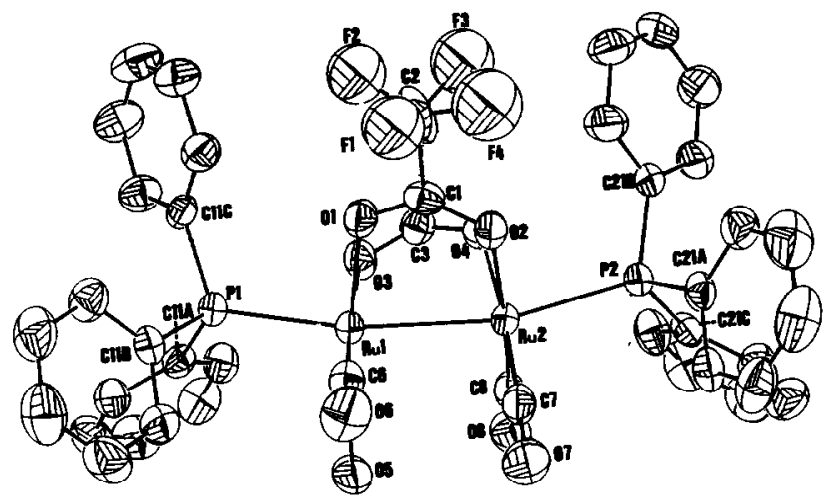

Fig. 3. ORTEP drawing of $\left[\mathrm{Ru}_{2}\left(\mathrm{O}_{2} \mathrm{CCF}_{3}\right)_{2}(\mathrm{CO})_{4}\left(\mathrm{PPh}_{3}\right)_{2}\right](3)$ with the numbering scheme. Thermal ellipsoids are drawn at the $50 \%$ probability level.

tances of $2.116,2.741 \AA$ in $2 ; 2.119,2.736 \AA$ in 1 ; and $2.149,2.728 \AA$ in 3 , respectively, it is apparent that the longer the $\mathrm{Ru}-\mathrm{O}$ distance, the weaker the negative coupling (and the shorter the $\mathrm{Ru}-\mathrm{Ru}$ distance). Thus, the observed mild inductive effect is probably due to the small range of $0.033 \AA$ in the Ru-O distances, if a dinuclear compound, $\left[\mathrm{Ru}_{2}\left(\mathrm{O}_{2} \mathrm{CR}\right)_{2}(\mathrm{CO})_{4}\left(\mathrm{PPh}_{3}\right)_{2}\right]$, can be prepared with rather long $\mathrm{Ru}-\mathrm{O}$ distances, the $\mathbf{R u}-\mathbf{R u}$ distance in this compound should be quite short and an appreciable inductive effect should be observed.

The Ru-P lengths of $2.4508(10) \AA$ in $1,2.4367(19)$, $2.4364(20) \AA$ in 2 , and $2.4430(21), 2.4492(21) \AA$ in 3 , are similar to each other and typical of the $\mathrm{Ru}-\mathrm{P}$ distances found in other mononuclear [26] or dinuclear [15] ruthenium complexes containing two phosphine groups, although the $\mathrm{Ru}-\mathrm{Ru}-\mathrm{P}$ angle of $163.539(23)^{\circ}$ in 1 is much smaller than those of $168.89(5), 166.91(5)^{\circ}$ in 2 and 168.11(6), 166.22(5) in 3. As reflected in the similar ${ }^{31} \mathrm{P}\left\{{ }^{1} \mathrm{H}\right\}$ NMR chemical shift around $20 \mathrm{ppm}$ observed for $\mathrm{PPh}_{3}$ in 1-3, the bonding interactions between the $\mathrm{Ru}^{\mathrm{I}}$ atoms and the phosphines of these compounds in solution should be very close to each other [27].

\section{Acknowledgment}

We wish to thank the National Science Council of the Republic of China for the financial support of this research (Contract No. NSC82-0208-M006-32).

\section{References}

1 K.-B. Shiu, K.-H. Yih, S.-L. Wang and F.-L. Liao, J. Organomet. Chem., 420 (1991) 359.

2 F. A. Cotton, in M. H. Chisholm (ed.), Inorganic Chemistry to- ward the 21st Century, ACS Symposium Series, No. 211, 1983, p. 209.

3 B. F. G. Johnson, (ed.), Transition Metal Clusters, Wiley, New York, 1980.

4 (a) F. A. Cotton and R. A. Walton, Multiple Bonds between Metal Atoms, John Wiley, New York, 1982; (b) F. A. Cotton and R. A. Walton, Struct. Bonding, 62 (1985) 1.

5 (a) M. Bianchi, G. Menchi, F. Francalanci, F. Piacenti, U. Matteoli, P. Frediani and C. Botteghi, J. Organomet. Chem., 188 (1980) 109; (b) M. Bianchi, P. Frediani, U. Matteoli, G. Menchi, F. Piacenti and G. Petrucci, J. Organomet. Chem., 259 (1983) 207; (c) U. Matteoli, G. Menchi, P. Frediani, M. Bianchi and F. Piacenti, J. Organomet. Chem., 285 (1985) 281.

$6 \mathrm{M}$. Rotem and Y. Shvo, Organometallics, 2 (1983) 1689.

7 G. Suss-Fink, G. Hermann, P. Morys, J. Elermann and A. Veit, J. Organomet. Chem., 284 (1985) 263.

8 J. Jenck, P. Kalck, E. Pinelli, E. Siani and A. Thorez, J. Chem. Soc., Chem. Commun., (1988) 1423.

9 P. Kalck, M. Siani, J. Jenck, B. Peyrille and Y. Peres, J. Mol. Catal., 67 (1991) 19.

10 J. G. Bullitt and F. A. Cotton, Inorg. Chim. Acta, 5 (1971) 406.

11 M. Spohn, T. Vogt and J. Stahle, Z. Naturforsch., 41B (1986) 1373.

12 (a) F. A. Cotton, M. W. Extine and L. D. Gage, Inorg. Chem., 17 (1978) 172; (b) F. A. Cotton, M. W. Extine and G. W. Rice, Inorg. Chem., 17 (1978) 176; (c) G. G. Christoph and Y.-B. Koh, J. Am. Chem. Soc., 101 (1979) 1422; (d) F. A. Cotton, W. H. Ilsley and W. Kaim, Inorg. Chem., 19 (1980) 1453; (e) F. A. Cotton and T. R. Felthouse, Inorg. Chem., 19 (1980) 323; (f) F. A. Cotton, W. H. Ilsley and W. Kaim, J. Am. Chem. Soc., 102 (1980) 3464; (g) B. E. Bursten and F. A. Cotton, Inorg. Chem., 20 (1981) 3042.

13 D. S. Bohle and H. Vahrenkamp, Inorg. Chem., 29 (1990) 1097.

14 K.-B. Shiu, C.-J. Chang, Y. Wang and M.-C. Cheng, J. Organomet. Chem., 406 (1991) 363.

15 H. Schumann, J. Opitz and J. Pickardt, J. Organomet. Chem., 128 (1977) 253.

16 M. Rotem, I. Goldberg, U. Shmueli and Y. Shvo, J. Organomet. Chem., 314 (1986) 185.

17 R. Mason, K. M. Thomas, D. F. Gill and B. L. Shaw, J. Organomet. Chem., 40 (1972) C67.

18 J. A. Cabeza, C. Landazuri, L. A. Oro, A. Tiripicchio and M. Tiripicchio-Camellini, J. Organomet. Chem., 322 (1987) C16.

19 S. J. Sherlock, M. Cowie, E. Singleton and M. M. de V. Steyn, J. Organomet. Chem., 361 (1989) 353.

20 M. M. de V. Steyn, E. Singleton, S. Hietkamp and D. C. Liles, J. Chem. Soc., Dalton Trans., (1990) 2991.

21 T. A. Albright, J. K. Burdett and M.-H. Whangbo, Orbital Interactions in Chemistry, Wiley, New York, 1985.

22 (a) R. H. Summerville and R. Hoffmann, J. Am. Chem. Soc., 98 (1976) 7240; (b) S. Shaik, R. Hoffmann, C. R. Fisel and R. H. Summerville, J. Am. Chem. Soc., 102 (1980) 4555.

23 (a) R. Mason and D. M. P. Mingo, J. Organomet. Chem., 50 (1973) 53. (b) B. K. Teo, M. B. Hall, R. F. Fenske and L. F. Dahl, Inorg. Chem., 14 (1975) 3103.

24 (a) J. G. Norman, Jr., H. J. Kolari, H. B. Gray and W. C. Trogler, Inorg. Chem., 16 (1977) 987; (b) J. G. Norman, Jr. and H. J. Kolari, J. Chem. Soc., Chem. Commun., (1975) 649; (c) J. G. Norman, Jr. and H. J. Kolari, J. Am. Chem. Soc., 97 (1975) 33.

25 (a) R. Hoffmann, Acc. Chem. Res., 4 (1971) 1; (b) P. J. Hay, J. C. Thibeault and R. Hoffmann, J. Am. Chem. Soc., 97 (1975) 4884.

26 G. Clark and S. M. James, J. Organomet. Chem., 134 (1977) 229.

27 P. S. Pregosin and R. W. Kunz, ${ }^{31} P$ and ${ }^{13} C$ NMR of Transition Metal Phosphine Complexes, Springer-Verlag, New York, 1979. 